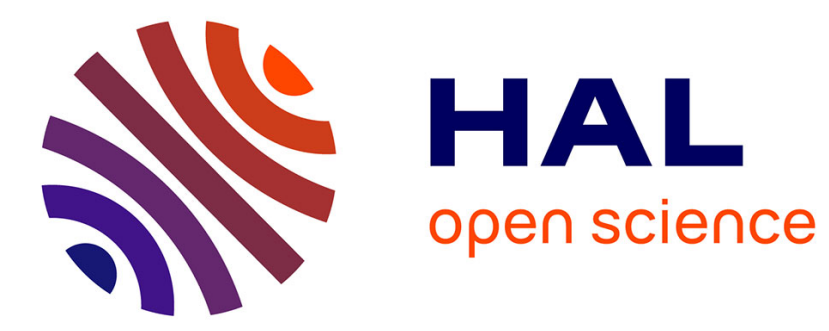

\title{
Chronique de droit pénal et de procédure pénale (juillet 2020 à décembre 2020)
}

Evelyne Bonis, Virginie Peltier

\section{To cite this version:}

Evelyne Bonis, Virginie Peltier. Chronique de droit pénal et de procédure pénale (juillet 2020 à décembre 2020). Titre VII - Les Cahiers du Conseil constitutionnel, 2021, No 6 (1), pp.96-109. 10.3917/tvii.006.0096 . hal-03461621

\section{HAL Id: hal-03461621 \\ https://hal.science/hal-03461621}

Submitted on 15 Dec 2021

HAL is a multi-disciplinary open access archive for the deposit and dissemination of scientific research documents, whether they are published or not. The documents may come from teaching and research institutions in France or abroad, or from public or private research centers.
L'archive ouverte pluridisciplinaire HAL, est destinée au dépôt et à la diffusion de documents scientifiques de niveau recherche, publiés ou non, émanant des établissements d'enseignement et de recherche français ou étrangers, des laboratoires publics ou privés. 


\section{Chronique de droit pénal et de procédure pénale (juillet 2020 à décembre 2020)}

\section{Évelyne BONIS}

Professeur à l'université de Bordeaux, Institut de sciences criminelles et de la justice (EA 4633)

\section{Virginie PELTIER}

Professeur à l'Université de Bordeaux, Institut de sciences criminelles et de la justice (EA 4633)

\section{I - PROCÉDURE PÉNALE}

\section{Décision $\mathrm{n}^{\circ}$ 2020-851/852 QPC du 3 juillet 2020,}

\section{Sofiane A. et autre [Habilitation à prolonger la durée des détentions provisoires dans un contexte d'urgence sanitaire]}

Dans cette décision, le Conseil constitutionnel était saisi de deux questions prioritaires de constitutionnalité, toutes deux transmises par la chambre criminelle de la Cour de cassation par arrêt en date du 26 mai 2020 dont il décide de procéder à la jonction pour y répondre par une seule décision (Cass. crim., 26 mai 2020, n 20-81.910 et n 20 81.971). La première portait sur la conformité du $2^{\circ}$ du paragraphe I de l'article 11 de la loi $n^{\circ} 2020-290$ du 23 mars 2020 d'urgence pour faire face à l'épidémie de covid-19 qui habilite le Gouvernement sur le fondement de l'article 38 de la Constitution, à prendre par ordonnance cer-taines mesures relevant en principe du domaine de la loi. Plus précisément, le $2^{\circ}$ de cet article prévoit qu'afin de faire face aux conséquences, notamment de nature administrative ou juridictionnelle, de la propagation de l'épidémie et des mesures prises pour lutter contre cette propagation, le Gouvernement peut prendre toute mesure adaptant les délais et procédures, les règles relatives au déroulement des instances ou encore adaptant, « aux seules fins de limiter la propagation de l'épidémie parmi les personnes participant aux procédures », les règles relatives au déroulement des mesures privatives de liberté avant jugement. Plus précisément, le d) de cet article instaure d'une part, une possibilité d'adapter les règles relatives au déroulement des gardes à vue, pour permettre l'intervention à distance de l'avocat et la prolongation de ces mesures pour au plus la durée légalement prévue sans présentation de la personne devant le magistrat compétent, et d'autre part, les règles relatives au déroulement et à la durée des détentions provisoires et des assignations à résidence sous surveillance électronique, pour permettre l'allongement des délais au cours de l'instruction et en matière d'audiencement, pour une durée proportionnée à celle de droit commun et ne pouvant excéder trois mois en matière délictuelle et six mois en appel ou en matière criminelle, et la prolongation de ces mesures au vu des seules réquisitions écrites du parquet et des observations écrites de la personne et de son avocat.

Les requérants, rejoints par certaines parties intervenantes, soutenaient que ces dispositions méconnaissaient l'article 66 de la Constitution qui fait de l'autorité judiciaire la gardienne des libertés et des droits de la défense. Par ailleurs, un des requérants reprochait au législateur de ne pas avoir suffisamment précisé, dans la loi d'habilitation, les conditions de cette prolongation automatique de la détention provisoire, ce qui serait constitutif d'une incompétence négative. Il faut dire que pour l'un des requérants, prévenu dans une procédure sui-vie contre lui des chefs d'assassinat, infraction à la législation sur les stupéfiants et association de malfaiteurs, cette prolongation automatique de la détention provisoire avait conduit la chambre de l'instruction à dire sans objet sa saisine aux fins de prolongations de la détention provisoire, ce que le prévenu contestait à l'occasion d'un pourvoi en cassation (pourvoi $\mathrm{n}^{\circ}$ 20-81.910). Pour l'autre requérant, prévenu dans une information suivie des chefs de tentative de meurtre en bande organisée, recel et destruction aggravée du bien d'autrui, un pourvoi en cassation avait été formé contre l'arrêt de la chambre de l'instruction confirmant l'ordonnance du juge des libertés et de la détention constatant la prolongation de plein droit de la détention provisoire (pourvoi $\mathrm{n}^{\circ} 20-81.971$ ). 
Ces questions prioritaires donnaient ainsi l'occasion au Conseil, non seulement d'apprécier la conformité à la Constitution du régime particulier de prolongation des détentions provisoires dans le contexte de la crise sanitaire, mais aussi, et pour la première fois, d'examiner, dans le cadre de son contrôle a posteriori, la conformité d'une loi d'habilitation ${ }^{1}$.

Au plan constitutionnel, on retiendra ainsi de cette décision, la nouveauté de ce contrôle qui est en réalité due à l'audace de la chambre criminelle de la Cour de cassation qui, à l'inverse du Conseil d'État, a décidé de transmettre au Conseil des questions portant sur les dispositions d'une loi d'habilitation ${ }^{2}$. Elle a, pour cela, considéré que de telles dispositions pouvaient faire l'objet d'un renvoi au Conseil constitutionnel dès lors que, par leur insuffisante précision, elles pouvaient porter atteinte à un droit ou une liberté constitutionnellement garanti. Le Conseil ainsi saisi a accepté d'examiner les dispositions d'une loi d'habilitation dans le cadre de son contrôle a posteriori, mais dans la stricte mesure de l'office que lui reconnaît l'article 61-1 de la Constitution ${ }^{3}$. Ainsi, après avoir reproduit les termes de cet article, il circonscrit étroitement le champ de son contrôle aux seuls griefs tirés de ce que les dispositions d'une loi d'habilitation portent atteinte, par elles-mêmes ou par les conséquences qui en découlent nécessairement, aux droits et libertés que la Constitution garantit (motif $n^{\circ} 9$ ).

Une fois reconnue cette possibilité d'un contrôle de constitutionnalité a posteriori des dispositions d'une loi d'habilitation, le Conseil constitutionnel se posa une autre question : celle de l'articulation de ce contrôle avec les dispositions d'une ordonnance prise sur le fondement d'une telle loi, car l'ar-ticle 61-1 ne l'investit que du pouvoir de vérifier la conformité d'une loi à la Constitution. Or, le propre des ordonnances prises par le Gouvernement dans le cadre d'une habilitation est d'avoir une valeur juridique évolutive. Alors que ces ordonnances sont adoptées par le Gouvernement et donc, à ce titre, n'ont qu'une valeur réglementaire, elles acquièrent ultérieurement valeur législative, une fois l'ordonnance ratifiée par le Parlement. De simple régiment, elles deviennent donc des lois et ceci à compter de la date de leur signature ${ }^{4}$. De cette évolution de la valeur de la norme, faut-il déduire que le Conseil constitutionnel peut contrôler un acte alors même qu'il n'est pas une loi parce qu'il est susceptible d'acquérir cette valeur ultérieurement ${ }^{5}$ ? Cette question a déjà reçu une réponse du Conseil qui, dans une décision n ${ }^{\circ} 2020-843$ QPC du 28 mai 20206, dont il reprend ici le sens, a jugé que «si les dispositions d'une ordonnance acquièrent valeur législative à compter de sa signature lorsqu'elles ont été ratifiées par le législateur, elles doivent être regardées, dès l'expiration du délai de l'habilitation et dans les matières qui sont du domaine législatif, comme des dispositions législatives au sens de l'article 61-1 de la Constitution. Leur conformité aux droits et libertés que la Constitution garantit ne peut donc être contestée que par une question prioritaire de constitutionnalité $»^{7}$.

\footnotetext{
1. À l'inverse, le contrôle de telles lois est désormais bien rôdé s'agissant de l'examen a priori de la constitutionnalité d'une loi d'habilitation avec de nombreuses décisions venues préciser ce contrôle. V. sur ce point, l'ensemble de la jurisprudence du Conseil retracée dans le commentaire de la décision du 3 juillet 2020 sur le site du Conseil : https://www.conseil-consti-tutionnel.fr/sites/default/files/as/root/bank_mm/ decisions/2020851_852qpc/2020851_852qpc_ccc.pdf
}

2. Contra : CE, 10e et 9e sous-sections réunies, 23 janv. 2015, n 380339 et plus récemment encore CE, 30 janvier 2019, Ordre des avocats à la cour d'appel de Paris et autres, $\mathrm{n}^{\circ}$ 408258, 408267, 408289, 408305, 415795, 415827 et 415901. Pour le Conseil d’État, " les dispositions législatives contestées se bornent à délimiter le champ de l'habilitation donnée au Gouvernement sur le fondement de l'article 38 de la Constitution pour prendre par ordonnance des mesures qui sont normalement du domaine de la loi ; qu'elles ne sont, dès lors, par leur nature même, pas susceptibles de porter atteinte aux droits et libertés que la Constitution garantit ».

3. A. Levade, « Ordonnance et loi d'habilitation. Le Conseil constitutionnel étend son empire », JCP, éd. G, 27 juill. 2020, n 30-35, pp. 14041405 .

4. C. Barthélemy, « Résurgence du débat sur la nature des ordonnances dans le domaine de la loi », RFDA, 2020, pp. 887-906.

5. G. Éveillard, «Les ordonnances de l'article 38 entre l'expiration du délai d'habilitation et la ratification législative... des dispositions législatives dépourvues de valeur législative », Droit administratif, novembre 2020, n 11, pp. 48-55; P. Deumier, « Les ordonnances non ratifiées ou la pyramide baroque », RTD civ. 2020, pp. 596-601.

6. Cons. const., déc. n 2020-843 QPC du 28 mai 2020, Force 5 (Autorisation d'exploiter une installation de production d'électricité)

7. Cons. 11. 
Au plan du régime de la détention provisoire, le Conseil fait porter son examen de conformité des mots « des détentions provisoires » figurant au d) du $2^{\circ}$ du paragraphe I de l'article 11 de la loi du 23 mars 2020 pour affirmer dans un premier temps que «les dispositions contestées n'excluent pas toute intervention d'un juge lors de la prolongation d'un titre de détention provisoire venant à expiration durant la période d'application de l'état d'urgence sanitaire » puis dans un second temps conclure que la loi telle qu'examinée ne porte atteinte ni par elle-même, ni par les conséquences qui en découlent nécessairement, aux exigences de l'article 66 de la Constitution imposant l'intervention d'un juge dans le plus court délai possible en cas de privation de liberté. Le Conseil conclut donc à la conformité de l'article de la loi à la Constitution, mais sans exclure pour autant une inconstitutionnalité qui pourrait résulter, non pas de la loi, mais de l'ordonnance, ce qui le conduit à préciser que « les dispositions d'une loi d'habilitation ne sauraient avoir ni pour objet ni pour effet de dispenser le Gouvernement, dans l'exercice des pouvoirs qui lui sont conférés en application de l'article 38 de la Constitution, du respect des règles et principes de valeur constitutionnelle, notamment les exigences résultant de son article 66 s'agissant des modalités de l'intervention du juge judiciaire en cas de prolongation d'une mesure de détention provisoire $»^{8}$. Il laissait ainsi sans doute entrevoir un possible motif de non-conformité à la Constitution.

Cette décision n'est ainsi que la première étape d'un long feuilleton relatif à la détention provisoire durant la crise sanitaire de la Covid 19 même si depuis, le contexte normatif a changé. La loi du 11 mai 2020 a certes prorogé l'état d'urgence sanitaire jusqu'au 10 juillet 2020 inclus, mais, s'agissant de la détention provisoire, elle a prévu des «modalités du retour progressif au droit commun $»^{9}$, de telle sorte que les prolongations sans intervention judiciaire sont restées limitées à la période du 25 mars 2020 au 11 mai 2020. En outre, et alors même que nous sommes de nouveau en état d'urgence sanitaire ${ }^{10}$, le Gouvernement n'a pas été, cette fois-ci, habilité à adapter par voie d'ordonnances les règles applicables à la prolongation des détentions provisoires.

S'agissant cependant des détentions prolongées entre mars et mai 2020, nous aurons l'occasion de revenir dans le prochain numéro sur les suites de ce feuilleton puisque le Conseil a dû de nou-veau connaître de ce contentieux à la suite du renvoi par la Cour de cassation, de deux nouvelles questions prioritaires de constitutionnalité relatives à la conformité aux droits et libertés que la Constitution garantit de l'article 16 de l'ordonnance $\mathrm{n}^{\circ}$ 2020-303 du 25 mars 2020 portant adaptation de règles de procédure pénale sur le fondement de la loi $\mathrm{n}^{\circ}$ 2020-290 du 23 mars 2020 d'urgence pour faire face à l'épidémie de covid-1911. Le contrôle ainsi demandé porte cette fois-ci directement sur l'article 16 de l'ordonnance, ce qui est désormais possible puisque cet article doit être regardé comme une disposition législative au sens de l'article 61-1 de la Constitution depuis l'expiration du délai de l'habilitation fixé au 24 juin 202012. Par une décision intervenue le 29 janvier 2021, le Conseil a déclaré cet article 16 contraire à la Constitution ${ }^{13}$.

$\mathrm{EB}$

8. Cons. 15

9. Art. 16-1 ajouté à l'ordonnance $\mathrm{n}^{\circ} 2020-303$ du 25 mars 2020.

10. V. décret $n^{\circ} 2020-1257$ du 14 octobre 2020 puis la prorogation opérée par la loi n 2020-1379 du 14 novembre 2020 autorisant la prorogation de l'état d'urgence sanitaire et portant diverses mesures de gestion de la crise sanitaire, jusqu'au 16 février 2021.

11. Cass. crim. 3 nov. 2020, pourvoi ${ }^{\circ} 20-83.189$

12. V. supra ce point et Cons. const., déc. n 2020-843 QPC du 28 mai 2020 ; Cons. const., décision n ${ }^{\circ}$ 2020-851/852 QPC du 3 juillet 2020.

13. Cons. const. déc. $n^{\circ}$ 2020-878/879 QPC du 29 janv. 2021, https://www.conseil-constitutionnel.fr/decision/2021/2020878_879QPC.htm 


\section{Décision $\mathbf{n}^{0}$ 2020-855}

QPC du 9 septembre 2020,

\section{Mme Samiha B. [Condition de paiement préalable pour la contestation des forfaits}

de post-stationnement]

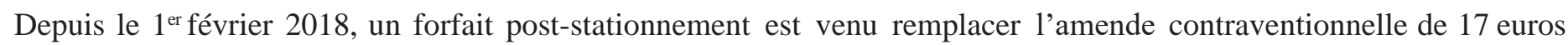
en cas de stationnement non payé ou insuffisamment réglé. Cette évolution résulte de la loi n $2014-58$ du 27 janvier 2014 relative à la modernisation de l'action publique territoriale et d'affirmation des métropoles (MAPTAM). Elle est le reflet d'une dépénalisation de la matière au profit de la mise en place d'une procédure à caractère administratif relevant de la compétence des collectivités territoriales. En vertu de l'article L. 2333-87 du Code général des collectivités territoriales, il est prévu que le conseil municipal ou l'organe délibérant de l'établissement public de coopération intercommunale ou du syndicat mixte compétent pour l'organisation de la mobilité peut instituer une redevance de stationnement, dont il détermine le barème tarifaire. Cette redevance doit être payée par le conducteur dès le début du stationnement. À défaut, l'intéressé s'expose à devoir s'acquitter d'un forfait de poststationnement, qui peut faire l'objet d'une majoration s'il n'est pas payé à temps. Les décisions individuelles relatives à ces forfaits et majorations peuvent être contestées. Le contribuable doit dans un premier temps effectuer un recours administratif préalable obligatoire dans le délai d'un mois à compter de la réception du forfait poststationnement.

Si ce recours est refusé en totalité ou en partie, un recours contentieux devant la Commission du contentieux du stationnement payant peut être engagé. L'article L. 2333-87-5 du Code général des collectivités territoriales subordonne toutefois la recevabilité de ce recours contentieux au paiement préalable du forfait de post-stationnement contesté et de sa majoration éventuelle. Ce sont ces conditions de recevabilité du recours contentieux que la requérante contestait dans la procédure transmise au Conseil constitutionnel et donnant lieu à la décision du 9 septembre $2020^{14}$.

En l'espèce, une personne avait reçu un titre exécutoire émis le 11 septembre 2018 par l'agence nationale de traitement automatisé des infractions en vue du recouvrement d'un forfait de post-stationnement mis à sa charge par la commune de Nancy. Par requête, elle avait sollicité auprès de la Commission du contentieux du stationnement payant l'annulation de ce titre. Par une ordonnance en date du 29 mai 2019, sa requête était rejetée. La requérante formait alors un pourvoi en cassation contre cette ordonnance devant le Conseil d'État. À l'occasion de ce recours, la demanderesse au pourvoi soulevait une question prioritaire de constitutionnalité portant sur la conformité à la Constitution des dispositions des II, IV et VI de l'article L. 2333-87 du Code général des collectivités territoriales, de celles de l'article L. 2333-87-5 du même code et de celles de l'article L. 2323-7-1 du Code général de la propriété des personnes publiques. Plus précisément, elle dénonçait une méconnaissance du droit à un recours juridictionnel effectif dans le fait de subordonner la recevabilité des recours contre les décisions individuelles mettant à la charge d'un justiciable un forfait de poststationnement au paiement préalable, par l'intéressé, du montant de ce forfait et de son éventuelle majoration, sans prévoir aucune exception. Par un arrêt rendu le 10 juin 2020, le Conseil d'État jugea la question suffisamment sérieuse pour opérer le renvoi de la question portant sur la conformité de l'article L. 2333-87-5 du Code général des collectivités territoriales, dans sa rédaction résultant de l'ordonnance ${ }^{\circ} 2015-401$ du 9 avril 2015 relative à la gestion, au recouvrement et à la contestation du forfait de post-stationnement pré- vus à l'article L. 2333-87 du Code général des collectivités locales au droit à un recours juridiction- nel effectif et aux droits de la défense tels qu'ils sont garantis par l'article 16 de la Déclaration des droits de l'homme et du citoyen du 26 août $1789^{15}$. Le Conseil constitutionnel statue sur cette ques- tion par sa décision du 9 septembre 2020, au terme d'un raisonnement en deux temps.

\footnotetext{
14. Sur cette décision : J.-A. Cano et L. Girard, « L'inconstitutionnalité de l'exigence du paiement préalable du forfait post-stationnement pour saisir la CCSP », La Semaine juridique. Administrations et collectivités territoriales, 21 septembre 2020, n 38-39, p. 2-3; J.-P. Céré, « Forfait de post-stationnement : le droit à un recours juridictionnel effectif s'impose », Actualité juridique. Pénal, novembre 2020, n 11, p. $521-522$.

15. CE, 10 juin 2020 , déc. $n^{\circ} 433276$.
} 
Dans un premier temps, le Conseil se prononce sur la possibilité qui est celle du législateur d'imposer des conditions à la recevabilité d'un recours eu égard au principe à valeur constitutionnelle du droit à un recours effectif qu'il a dégagé de l'article 16 de la Déclaration des droits de l'homme et du citoyen. Il énonce à cet égard que le législateur peut valablement subordonner le recours à des conditions telles que le paiement d'un forfait. Il y voit en effet un moyen à la disposition du législateur pour prévenir les recours dilatoires dans un contentieux exclusivement pécuniaire susceptible de concerner un très grand nombre de personnes. Si restriction il y a au droit d'accès à un tribunal, celle-ci est justifiée au nom de la bonne administration de la justice. De ce point de vue, la décision n'est pas sans rappeler, en matière pénale, la position de la Cour européenne des droits de l'homme qui, au sujet du contentieux des recours contre les amendes forfaitaires en matière de contravention, jugeait, au visa de l'article 6 de la Convention de sauvegarde des droits de l'homme et des libertés fondamentales que l'article 529-10 du Code de procédure pénale qui subordonne le droit d'accès au tribunal de police au paiement préalable d'une somme dont le montant est égal à celui de l'amende forfaitaire est une restriction au droit d'accès imposée dans le but d'assurer une bonne administration de la justice ${ }^{16}$. Elle estimait ainsi la restriction légitime compte tenu du but poursuivi par cette obligation de consignation, à savoir prévenir l'exercice de recours dilatoires et abusifs et éviter l'encombrement excessif du rôle du tribunal dans le domaine de la circulation routière, qui concerne l'ensemble de la population et se prête à des contestations fréquentes.

Cependant, dans un second temps, le Conseil s'attache à vérifier que, dans ses conditions de mise en œuvre, ce système présente des garanties de nature à s'assurer que l'exigence d'un paiement préalable ne porte pas une atteinte substantielle au droit d'exercer un recours juridictionnel effectif. Or, selon lui, deux éléments sont de nature à priver le dispositif de forfait post-stationnement, de ces garanties. D'une part, il constate que si, conformément à l'article L. 2333-87 du Code général des collectivités territoriales, le montant du forfait de post-stationnement ne peut excéder celui de la redevance due, aucune disposition législative ne garantit que la somme à payer ne soit pas d'un montant trop élevé. D'autre part, il observe que le législateur n'a apporté à l'exigence de paiement préalable desdits forfaits et majorations aucune exception tenant compte de certaines circonstances ou de la situation particulière de certains redevables. Il déduit de ces deux considérations que le législateur n'a pas prévu les garanties de nature à assurer que l'exigence de paiement préalable ne porte pas d'atteinte substantielle au droit d'exercer un recours juridictionnel effectif. Il déclare donc contraires à la Constitution les dispositions contestées. Cette déclaration d'inconstitutionnalité est applicable à toutes les affaires non jugées définitivement à la date de la décision. Cette décision invite donc à apprécier les modalités de recours et le droit à un recours juridictionnel effectif à l'aune de la situation des justiciables, ce sur quoi le Défenseur des droits avait pu alerter ${ }^{17}$. Là encore, ces arguments font écho au pénaliste au sujet de la procédure de l'amende forfaitaire dont le législateur a récemment étendu le champ d'application en matière correctionnelle. De façon assez comparable à ce que prévoit le Code des collectivités territoriales pour le forfait post-stationnement, dans la procédure de l'amende forfaitaire, tant en matière contraventionnelle que correctionnelle, si l'agent dispose de voies de droit pour contester l'amende forfaitaire devant un juge ${ }^{18}$, les textes subordonnent la recevabilité du recours à une consignation préalable dont le montant est égal à celui de l'amende forfaitaire dans le cas d'une requête en exonération et à celui de l'amende majorée dans le cas d'une réclamation ${ }^{19}$. Saisie d'une requête en la matière, la Cour européenne des droits de l'homme s'en remet certes à la marge d'appréciation reconnue aux États quant aux conditions de recevabilité d'un recours, mais, dans l'appréciation des faits de chaque espèce, elle intègre aussi à son analyse la situation exacte du requérant, ce qui la conduit à apprécier si une entrave disproportionnée au droit d'accès à un tribunal est portée en ayant égard aux ressources de la personne ${ }^{20}$.

16. CEDH, 29 avr. 2008, Thomas c/ France, req. n 14279/05: RSC 2008. 697, obs. D. Roets. Dans le même sens : CEDH, 30 juin 2009 , n 49852/06, Schneider c/ France, D. 2009. 2110, obs. S. Lavric ; ibid. 2771, obs. J.-F. Renucci.

17. Défenseur des droits, «La défaillance du forfait de post-stationnement : rétablir les droits des usagers », rapport 2020.

18. V. au sujet de la conformité de l'article 529-10 du Code de procédure pénale à l'article 16 de la Déclaration des droits de l'homme : Cons. const., déc. n 2010-38 QPC du 29 sept. 2010 ayant conduit le Conseil à s’interroger sur la question de savoir si le pouvoir du parquet était ou non conforme au droit à un procès équitable, le requérant soulignant qu'il n'existe pas de voie de recours aménagée contre la décision par laquelle l'officier du ministère public rejette pour irrecevable une requête en exonération de la conformité ; Cons. const., déc. n² 2010-38 QPC du 29 sept. 2010, AJ pénal, 2010. 555, obs. J.-P. Céré ; RSC, 2011. 187, obs. B. de Lamy ; Dr. Pénal, 2010, n 120, obs. J.-H. Robert.

19. Code de procédure pénale, art. 495- 17 et s. en matière correctionnelle et art. 529 et s. en matière contraventionnelle.

20. CEDH, 30 juin 2009, n 49852/06, Schneider c/ France, op. cit. 
De la confrontation des décisions du Conseil constitutionnel du 9 septembre 2020 et de la Cour européenne, peut donc se poser la question de la conformité des textes relatifs aux recours contre les amendes forfaitaires, car, tant en matière contraventionnelle que correctionnelle, la prise en considération « de certaines circonstances ou de la situation particulière de certains redevables » est minimale même si elle n'est pas totalement absente. On relèvera ainsi que l'article 529-10, $2^{\circ}$, du Code de procédure pénale prévoit, s'agissant de la contestation d'une amende forfaitaire contraventionnelle, qu'il est possible d'échapper à la consignation en produisant soit le récépissé du dépôt de plainte pour vol ou destruction du véhicule ou pour le délit d'usurpation de plaque d'immatriculation prévu par l'article L. 317-4-1 du Code de la route, ou une copie de la déclaration de destruction de véhicule établie conformément aux dispositions du Code de la route, soit une lettre signée de l'auteur de la requête ou de la réclamation précisant l'identité, l'adresse, ainsi que la référence du permis de conduire de la personne qui était présumée conduire le véhicule lorsque la contravention a été constatée ; soit des copies de la déclaration de cession du véhicule et de son accusé d'enregistrement dans le système d'immatriculation des véhicules. De façon comparable, en matière correctionnelle, l'article 495-20 du Code de procédure pénale exonère également de la consignation préalable le prévenu qui produira un récépissé de dépôt de plainte pour le délit d'usurpation d'identité prévu à l'article 434-23 du Code pénal. Si ces textes introduisent ainsi bien des éléments permettant de prendre en considération « certaines circonstances ou de la situation particulière de certains redevables » comme le souhaite le Conseil, rien n'est, en revanche, prévu dans le Code de procédure pénale s'agissant de la situation patrimoniale des prévenus de telle sorte qu'à l'aune de la décision de la CEDH précitée de 2009 comme de la décision du Conseil du 9 septembre 2020, des doutes peuvent subsister sur la conformité des articles 529-10 et 495-20 du Code de procédure pénale à la Constitution ${ }^{21}$. Ces doutes sont d'autant plus grands aujourd'hui que la procédure de l'amende forfaitaire a été étendue par l'effet de la loi du 23 mars 2019 à de nombreux délits pour lesquels les amendes encourues sont parfois élevées ${ }^{22}$ - et donc les consignations préalables au recours importantes - des montants sans commune mesure avec les forfaits de post-stationnement. Le fait qu'en cas de relaxe, le montant de la consignation soit reversé à la personne ne change rien au problème dès lors que la consignation de telles sommes est une condition de recevabilité du recours et donc d'accès au juge ${ }^{23}$.

EB

21. Sur cette question, E. Bonis, « La forfaitisation de la réponse pénale : la simplification au mépris des droits fondamentaux ? » in $L a$ simplification de la procédure pénale, Actes d'un colloque tenu à Corte le 23 mars 2018, PUAM 2019, dir. Y. Carpentier et A. Guidicelli, p. 83 à 100

22. Selon l'article 495-17 du Code de procédure pénale renvoyant à l'article 131-13 du Code pénal, l'amende peut atteindre 3000 euros. À titre d'illustration, pour l'infraction prévue à l'article L. 3315-5 alinéa 3 du Code des transports, le montant de l'amende forfaitaire majorée est de 1600 euros. En matière d'infractions au Code de la santé publique, il est de 450 voire 600 euros selon les infractions (C. santé publ., art. L. 3352-5 ; 3353-3 et 3421-1, al. 3).

23. Au sujet de ce reversement, C. pr. pén., art. 495-21, al. 3. 


\section{Décision $n^{\circ}$ 2020-858/859}

\section{QPCdu2 octobre2020.}

\section{Détention provisoire. Conditions d'incarcération des détenus (JCP G 2020, $1348 ;$ RDPD 2020, $n^{\circ} 4$ à paraître), M. Geoffrey F. et autre [Conditions d'incarcération des détenus]}

Le 2 octobre 2020, le Conseil constitutionnel a mis un terme au volet jurisprudentiel relatif à la prise en compte des conditions d'exécution d'une détention provisoire. La question qui se posait était effectivement de savoir si un détenu pouvait exciper de conditions de détention contraires à la dignité pour obtenir une mainlevée de sa détention provisoire. La question était d'importance puisque cette privation de liberté, régie par les articles 144 et suivants du Code de procédure pénale, s'effectue dans des maisons d'arrêt, c'est-à-dire dans des établissements vétustes, souvent insalubres, où la surpopulation carcérale atteint des niveaux plus que préoccupants.

Ce mouvement prétorien de réflexion a été initié par un arrêt de la Cour européenne des droits de l'homme du 30 janvier 2020 qui a condamné la France pour violation, d'une part, de l'article 3 de la Convention de sauvegarde des droits de l'homme et des libertés fondamentales - les conditions de détention dans plusieurs établissements pénitentiaires (centres pénitentiaires, maisons d'arrêt) ayant été jugées constitutives de traitements inhumains et dégradants - et, d'autre part, de l'article 13, la Cour estimant que les détenus ne bénéficiaient pas, en l'état du droit positif, d'un recours juridictionnel effectif de nature à mettre fin à ces conditions de détention indignes (CEDH, 30 janv. 2020, n ${ }^{\circ} 9671 / 15$ et 31 autres, JMB c. France : JCP G 2020, 154, aperçu rapide B. Pastre-Belda ; Rev. pénit. 2020, n 1, p. 179, obs. C. Margaine). On notera, malgré tout, que, dans un arrêt Barbotin c. France du 19 novembre 2020, la Cour européenne a modifié sa position en raison de l'existence d'un recours indemnitaire devant le tribunal administratif, même si elle a conclu à une nouvelle violation de l'article 13, le détenu s'étant retrouvé, en l'espèce, débiteur de l'État français à la suite du recours qu'il avait intenté (CEDH, 19 nov. 2020, Barbotin c. France, n 25338/16 : Dr. pén. 2021, comm. 19). Toujours est-il que la Cour de cassation s'est emparée de la question, dans deux arrêts du 8 juillet 2020, pour créer de toutes pièces un recours au profit des détenus provisoires, en s'appuyant sur la décision européenne de janvier aux termes de laquelle, à partir du moment où le requérant a fait une description précise et crédible de ses conditions de détention, la charge de la preuve doit alors être reportée sur l'État défendeur qui reste le mieux à même d'avoir accès à toutes les informations pour confirmer ou, à l'inverse, infirmer les allégations du détenu, en fournissant à son tour une description détaillée de ses conditions de détention (CEDH, 30 janv. 2020, JMB c. France, préc., $\mathrm{n}^{\circ}$ 258). Ainsi, la chambre criminelle a-t-elle mis au jour un mécanisme permettant au détenu provisoire d'alerter les autorités judiciaires de l'indignité de ses conditions de détention : "lorsque la description faite par le demandeur de ses conditions personnelles de détention est suffisamment crédible, précise et actuelle, de sorte qu'elle constitue un commencement de preuve de leur caractère indigne, il appartient alors à la chambre de l'instruction, dans le cas où le ministère public n'aurait pas préalablement fait vérifier ces allégations, et en dehors du pouvoir qu'elle détient d'ordonner la mise en liberté de l'intéressé, de faire procéder à des vérifications complémentaires afin d'en apprécier la réalité. Après que ces vérifications ont été effectuées, dans le cas où la chambre de l'instruction constate une atteinte au principe de dignité à laquelle il n'a pas entre-temps été remédié, elle doit ordonner la mise en liberté de la personne, en l'astreignant, le cas échéant, à une assignation à résidence avec surveillance électronique ou à un contrôle judiciaire » (Cass. crim., 8 juill. 2020, $\mathrm{n}^{\circ} 20-81.731$ et $\mathrm{n}^{\circ} 20-81.739$ : JCP G 2020, 1075).

C'est dans ce contexte que le Conseil constitutionnel a été conduit à intervenir puisque, par les arrêts du 8 juillet 2020, la Cour de cassation lui avait transmis deux questions prioritaires de constitutionnalité portant sur la conformité aux principes de sauvegarde de la dignité de la personne humaine, de prohibition des traitements inhumains et dégradants, de liberté individuelle, de droit à un recours juridictionnel effectif et de droit au respect de la vie privée, des articles 137-3 du Code de procédure pénale (dans sa rédaction résultant de la loi du 23 mars 2019), 144 du même code (dans sa rédaction résultant de la loi du 24 novembre 2009) et 144-1 du même code (dans sa rédaction résultant de la loi du 15 juin 2000) qui n'imposeraient pas au juge judiciaire de faire cesser des conditions de détention provisoire contraires à la dignité de la personne humaine. 
Le Conseil, restreignant le domaine de la question, limite son examen au seul alinéa 2 de l'article 144-1 du Code de procédure pénale, selon lequel le juge d'instruction ou, s'il est saisi, le juge des libertés et de la détention, doit ordonner la mise en liberté immédiate de la personne placée en détention provisoire, selon les modalités prévues par l'article 147, dès que les conditions prévues à l'article 144 ne sont plus remplies. Il abroge cette disposition avec effet différé au $1^{\text {er }}$ mars 2021, retenant une méconnaissance des articles 9 (droit au respect de la présomption d'innocence) et 16 (droit à un recours juridictionnel effectif) de la Déclaration des droits de l'homme et du citoyen. Son analyse est double, portant autant sur les modalités de son contrôle que sur le fond du droit.

En premier lieu, le Conseil se voit dans l'obligation de répondre aux arguments du Premier ministre qui soutenait que, dans la mesure où la Cour de cassation - par ses arrêts du 8 juillet 2020 - a mis le droit interne en conformité avec les solutions conventionnelles, son contrôle de constitutionnalité ne s'imposait plus. Sans même entrer dans des considérations de droit constitutionnel, la saugrenuité de cette logique ne peut manquer de susciter l'interrogation au regard des conséquences qu'elle entraîne : elle prive le Conseil d'exercer un contrôle qui lui a été demandé par un requérant, donc un justiciable (qui plus est privé de liberté avant jugement, raison pour laquelle la décision est rendue sur le fondement de l'article 9 DDHC garantissant le respect de la présomption d'innocence) et abstrait le législateur de sa compétence - mais aussi de sa responsabilité - d'instaurer les règles de droit nécessaires, au seul motif (prétexte ?) que la Cour de cassation aurait déjà réglé la question. Là encore, le principe de la légalité criminelle, déjà fortement malmené par la chambre criminelle, est totalement ignoré du législateur qui croit bon de s'en remettre à des magistrats qui, si haut placés soient-ils dans la hiérarchie judiciaire, ne sont élus par personne, à l'inverse des députés censés représenter le peuple français. Toujours est-il que le Conseil va devoir justifier son intervention, rappelant que la Constitution se trouvant au sommet de la hiérarchie des normes, il ne lui appartient pas de tenir compte de l'interprétation qui aurait été faite d'une disposition (dont la contrariété à la Constitution est soulevée) par une juridiction pour la rendre conforme aux engagements internationaux de la France. En d'autres termes, les contrôles de conventionnalité de la Cour de cassation et de constitutionnalité qui lui revient sont autonomes et ne peuvent avoir de répercussions l'un sur l'autre. Le lecteur attentif s'en était aperçu puisque le contrôle de constitutionnalité par lequel le Conseil avait abrogé la quasi-totalité du régime juridique de la garde à vue (Cons. const., déc. n 2010-14/22 QPC du 30 juillet 2010) n'avait pas empêché l'assemblée plénière de la Cour de cassation de prendre position dans ses arrêts du 15 avril 2011 pour répondre aux exigences européennes en la matière (CEDH, Salduz c. Turquie, 27 nov. 2008, n ${ }^{\circ}$ 36391/01; CEDH, Dayanan c. Turquie, 13 oct. 2009, n 7377/03), au motif que les États adhérents à la Convention de sauvegarde des droits de l'homme et des libertés fondamentales doivent respecter les décisions de la Cour euro- péenne sans attendre d'être attaqués devant elle ni d'avoir modifié leur législation (Ass. plén., 15 av. 2011, $\mathrm{n}^{\circ} 10-30.316$, $\mathrm{n}^{\circ}$ 10-17.049, $\mathrm{n}^{\circ}$ 10-30.242, $\mathrm{n}^{\circ}$ 10-30.313). En l'espèce, le mouvement était inverse puisque la décision de la Cour de cassation avait précédé l'intervention du Conseil, mais le résultat est le même : chacun se livre au contrôle pour lequel il a été saisi.

Précisément, en second lieu, le Conseil choisit d'abroger l'alinéa 2 de l'article 144-1 du Code de procédure pénale après avoir remarqué qu'aucun recours ne permet au juge judiciaire de remettre en liberté un détenu qui exécuterait sa détention provisoire dans des conditions indignes, conclusion à laquelle la Cour européenne des droits de l'homme était déjà parvenue le 30 janvier 2020 (voir aussi, pour une absence de recours, en matière pénale, CEDH, 21 mai 2015, Yengo c. France, no 50494/12). En effet, le texte ne prévoit de possibilité de mainlevée de la détention que lorsque les conditions mentionnées à l'article 144 du même Code (ou à l'article 144-1 qui précise que la détention provisoire ne peut excéder une durée raisonnable, au regard de la gravité des faits reprochés à la personne mise en examen et de la complexité des investigations nécessaires à la manifestation de la vérité) ne sont plus remplies. Or, ces dernières ne font référence qu'aux objectifs poursuivis par la détention provisoire - insusceptibles par ailleurs d'être satisfaits par un placement sous contrôle judiciaire ou par une assignation à résidence sous surveillance électronique - comme, par exemple, conserver les preuves ou les indices matériels qui sont nécessaires à la manifestation de la vérité ou empêcher une pression sur les témoins ou les victimes ainsi que sur leur famille (CPP, art. $1441^{\circ}$ et $2^{\circ}$ ). La situation est logique, la loi ne se bornant qu'à organiser les modalités d'une mesure nécessaire à la manifestation de la vérité judiciaire, dont il est sous-entendu qu'elle s'exécute dans le respect de la dignité humaine. Or, les conditions d'hébergement dans certains établissements pénitentiaires sont telles que cette condition de dignité pour le moins évidente, et qui n'a, de ce fait, pas sa place dans le régime juridique d'une mesure procédurale, a néanmoins fini par s'y imposer. 
C'est en tout cas la ligne de conduite tracée par le Conseil constitutionnel au législateur puisqu'il redéfinit les rôles du juge et du Parlement : s'il incombe " aux autorités et juridictions compétentes, de prévenir et de réprimer les agissements portant atteinte à la dignité de la personne placée en détention provisoire et d'ordonner la réparation des préjudices subis », il revient "au législateur de garantir aux personnes placées en détention provisoire la possibilité de saisir le juge de conditions de détention contraires à la dignité de la personne humaine, afin qu'il y soit mis fin ». Le législateur va donc devoir réécrire l'article 144-1, alinéa 2 du Code de procédure pénale, quoique le texte à modifier soit plutôt l'article 144 puisque c'est lui qui énumère les cas dans lesquels la mainlevée de la détention provisoire peut être ordonnée.

Deux remarques à titre de conclusion.

Tout d'abord, on peut s'interroger sur la cohérence de cette décision lorsqu'on la met en contemplation de celle rendue le 29 septembre 2010, toujours en matière de droit à un recours juridictionnel effectif, mais au sujet cette fois de l'amende forfaitaire (Cons. const., déc. $n^{\circ}$ 2010-38 QPC du 29 sept. 2010). En effet, alors que l'article 529-10 du Code de procédure pénale n'offrait aucun recours au justiciable condamné au paiement d'une amende forfaitaire, contre une décision d'irrecevabilité de sa requête en exonération émanant du parquet, le Conseil, après avoir imposé la création d'un recours par une réserve d'interprétation - à laquelle la Cour européenne des droits de l'homme avait été sensible (CEDH, 8 mars 2012, Cadène c. France, n 12039/08 ; CEDH, 8 mars 2012, Célice c. France, n 14166/09) tout comme d'ailleurs la Cour de cassation qui avait précisé que la décision devait pouvoir faire l'objet d'un recours devant la juridiction de proximité (Cass. crim., 25 mars 2014, n 13-80.170, Bull. crim. n 93) - n'avait pas abrogé le texte. Mais, le 2 octobre 2020, il le fait pour la détention provisoire, alors qu'un recours avait été mis en place par la Cour de cassation.

Ensuite, cette décision ne pouvait qu'ouvrir la voie à d'autres contestations, comme l'atteste la décision par laquelle le Conseil d'État a choisi de renvoyer au Conseil constitutionnel une question prioritaire de constitutionnalité qui soulève l'inconstitutionnalité de nombreux aménagements de peines, au motif que les textes qui les régissent n'offrent pas la possibilité au juge de l'application des peines de tirer les conséquences de conditions de détention contraires à la dignité afin qu'il y soit mis fin (CE, 27 janv. 2021, n 445873). L'épilogue offert par le Conseil à la question de la détention provisoire n'aura donc été qu'un prologue en matière de peine. À suivre, donc... 


\section{Décision $n^{\circ}$ 2020-805 DC du 7 août 2020. Terrorisme. Inconstitutionnalité des mesures de sûreté à l'encontre des auteurs d'infractions terroristes à l'issue de leur peine (Dr. pén. 2010, comm.182; Dr.pén.2020, étude 30, par M. Brenaut).}

Avant même d'avoir vu le jour, la loi n 2020-1023 du 10 août 2020 instaurant des mesures de sûreté à l'encontre des auteurs d'infractions terroristes à l'issue de leur peine, avait perdu l'essentiel de sa substance puisque le Conseil constitutionnel, dans une décision du 7 août 2020, a abrogé la totalité du dispositif proposé, à savoir les articles 706-25-15 et suivants que la proposition de loi du 27 juillet 2020 souhaitait intégrer dans le titre XV relatif à la poursuite, à l'instruction et au jugement des actes de terrorisme du livre IV du Code de procédure pénale. La raison de l'intervention législative trouvait sa source dans la volonté de réagir à des chiffres rendus publics par la Chancellerie, selon lesquels 153 personnes détenues pour des faits de terrorisme seraient libérées dans les trois années à venir. Il s'agissait donc ici d'organiser un dispositif de surveillance post-sentenciel, inspiré de la surveillance judiciaire (CPP, art. 723-29 et suivants), à destination des personnes condamnées pour une infraction terroriste (C. pén., art. 421-1 à 421-6) - à l'exclusion de la provocation au terrorisme et de l'apologie de celui-ci -, à une peine privative de liberté d'une durée d'au moins cinq ans ou, en cas de récidive légale, de trois ans minimum. Pour être astreinte à la mesure, la personne devait présenter, à la fin de l'exécution de sa peine, une particulière dangerosité caractérisée, d'une part, par une probabilité très élevée de récidive et, d'autre part, par une adhésion persistante à une idéologie ou à des thèses incitant à la commission d'actes de terrorisme. Le régime juridique de la surveillance prévoyait alors l'obligation de respecter un certain nombre d'obligations, certaines susceptibles d'être imposées à tout type de condamné (répondre aux convocations du juge de l'application des peines ou du service pénitentiaire d'insertion et de probation ou recevoir les visites de ce service et lui communiquer les renseignements ou documents de nature à permettre le contrôle de ses moyens d'existence et de l'exécution de ses obligations, par exemple), d'autres en rapport plus étroit avec les faits commis (comme respecter les conditions d'une prise en charge sanitaire, sociale, éducative ou psychologique, destinée à permettre sa réinsertion et l'acquisition des valeurs de la citoyenneté, le cas échéant au sein d'un établissement d'accueil adapté dans lequel elle aurait été tenue de résider). La mesure était d'une durée maximale d'un an, renouvelable pour la même durée dans la limite de 5 ans (ou 10 en cas de crime ou de délit passible de 10 ans d'emprisonnement), ces plafonds étant réduits à 3 et 5 ans en cas de minorité du condamné. La décision de placement devait être prise par la juridiction régionale de la rétention de sûreté de Paris (le tribunal pour enfants de Paris pour les mineurs), au vu d'un avis motivé de la commission pluridisciplinaire des mesures de sûreté chargée d'évaluer la dangerosité de la personne.

Les griefs soulevés par les députés et sénateurs auteurs de la saisine étaient fort nombreux puisqu'il était reproché à l'article 1er instaurant la mesure de sûreté, d'entraver la liberté personnelle par une rigueur non nécessaire et de porter à la liberté individuelle, à la liberté d'aller et de venir et au droit au respect de la vie privée une atteinte qui n'était ni nécessaire, ni adaptée, ni proportionnée à l'objectif poursuivi par le législateur, mais encore de méconnaître le principe de légalité des délits et des peines en raison de la subjectivité de l'appréciation de la dangerosité de la personne et de conférer à la mesure contestée, du fait du cumul de certaines obligations, un caractère privatif de liberté justifiant l'application du principe de non-rétroactivité de la loi pénale. Le projet étant contesté jusque dans le propre camp de l'exécutif, le président de l'Assemblée nationale demandait également au Conseil constitutionnel d'examiner la conformité de ces dispositions à l'article 9 de la Déclaration des droits de l'homme et du citoyen de 1789 et à l'article 66 de la Constitution. Le Conseil statue uniquement sur le fondement de l'article 9 garantissant le respect de la présomption d'innocence et commence par examiner la nature de la mesure pour en tirer un certain nombre de conséquences. En premier lieu, il met en exergue différents éléments qui vont lui permettre de conclure qu'elle n'est ni une peine ni une sanction ayant le caractère d'une punition : elle est, en effet, prononcée en considération d'une condamnation pénale et succède à l'accomplissement de la peine. Elle n'est pas décidée par la juridiction de jugement, mais par la juridiction régionale de la rétention de sûreté, une fois la peine exécutée. Ayant pour but de prévenir la récidive, elle repose non sur la culpabilité de la personne condamnée, mais sur sa particulière dangerosité, appréciée à la date de la décision. On retrouve dans cette énumération les éléments déjà mis en exergue par le Conseil pour exclure toute existence d'une peine ou d'une mesure punitive dans ses décisions du 8 décembre 2005 relative à la surveillance judiciaire et au placement sous surveillance électronique mobile et du 21 février 2008 sur la rétention et la surveillance de sûreté (Cons. const., déc. n 2005-527 DC du 8 déc. 2005 ; Cons. const., déc. n 2008-562 DC du 21 fév. 2008). 
Le raisonnement développé est alors le suivant qui pose, en quelque sorte, les conditions requises pour qu'un nouveau dispositif de surveillance voie le jour : la mesure n'est donc pas punitive, mais elle doit malgré tout respecter le principe de nécessité selon lequel la liberté personnelle ne saurait être entravée par une rigueur qui ne serait pas nécessaire. L'appréciation de cette nécessité découle alors de la mise en balance d'un certain nombre d'éléments : d'une part, la mesure s'ajoute à d'autres qui existent déjà (surveillance judiciaire, suivi sociojudiciaire, rétention de sûreté, surveillance de sûreté, fichier national des auteurs d'infractions terroristes (FNAIT), sans compter les MICAS, mesures individuelles de contrôle administratif et de surveillance des articles L228-1 à L228-7 du Code de la sécurité intérieure) et, d'autre part, elle vise à répondre au risque particulier de récidive que présente une personne qui persiste à adhérer, à la fin de sa peine, à une idéologie prônant la commission d'actes de terrorisme. Il incombe de ce fait au législateur d'assurer une conciliation entre la protection de l'ordre public (le terrorisme le troublant gravement par l'intimidation et la terreur) et l'exercice des droits et libertés de chacun, y compris des condamnés pour terrorisme. En résumé, pour qu'un dispositif nouveau puisse voir le jour, il est indispensable qu'il soit à la fois subsidiaire (aucune mesure existante ne doit pouvoir être appliquée) et proportionné à l'objectif de protection de l'ordre public.

Pour conclure à l'inconstitutionnalité de l'article 1er, 2 et 4 du projet de loi, le Conseil s'appuie sur cinq arguments qu'il développe à l'aune des conditions préalablement mises au jour, certaines démentant sa subsidiarité (la mesure de sûreté peut être prononcée même lorsque la peine a été assortie d'un sursis, y compris simple et il n'est pas exigé que le condamné ait pu, pendant l'exécution de sa peine, bénéficier de mesures destinées à faciliter sa réinsertion), d'autres sa proportionnalité (la durée maximale est fonction de la peine encourue et non de la peine prononcée, son renouvellement peut être décidé aux mêmes conditions que la décision initiale, sans qu'il soit exigé que la dangerosité de la personne soit corroborée par des éléments nouveaux ou complémentaires), alors que les obligations et interdictions qui en découlent portent atteinte aux droits et libertés.

\section{Ce nouveau dispositif était-il véritablement surabondant?}

Tout d'abord, il est vrai qu'il ressemblait beaucoup à la surveillance judiciaire, mécanisme de contrôle post-sentenciel reposant sur le constat de la dangerosité du condamné et assorti des obligations du sursis probatoire, comme la plupart des obligations que la nouvelle mesure imposait. De même, pouvait-elle s'apparenter à un suivi socio-judiciaire supplémentaire puisque le suivi des articles 131-36-1 et suivants du Code pénal, applicable aux terroristes depuis la loi du 3 juin 2016, est devenu une peine complémentaire obligatoire par l'effet de la loi du 10 août 2020 qui visait donc à installer cette surveillance et que cette dernière comprenait les mêmes obligations que le suivi socio-judiciaire, qui offrait, quant à lui, l'avantage de pouvoir être prononcé pour une durée plus longue (10 ans en matière correctionnelle, 20 ans pour les délits punis de 10 ans d'emprisonnement, 20 ans pour les crimes, cette durée pouvant être de 30 ans ou même perpétuelle selon les cas). Mais il présentait l'inconvénient majeur de ne pas pouvoir être renouvelé et, surtout, de ne pas s'appliquer aux auteurs d'infractions commises avant l'entrée en vigueur de la loi du 3 juin 2016, même si ce « défaut » pouvait être compensé par leur inscription au FNAIT ou par l'application des MICAS, qui comprennent des obligations plus rigoureuses (à l'image de l'assignation à résidence), mais d'une durée plus courte (un an maximum). Ensuite, il n'était pas exigé que le condamné ait fait l'objet, au cours de l'exécution de sa peine, de mesures de nature à favoriser sa réinsertion. Pourtant, la question de la prise en charge des condamnés tout au long de l'exécution de leur peine est devenue centrale sous l'impulsion de la jurisprudence de la Cour européenne des droits de l'homme qui n'hésite plus à conclure à une violation des dispositions de la Convention de sauvegarde des droits de l'homme et des libertés fondamentales en son absence. Ainsi, la Cour, dans sa décision M. c. Allemagne du 12 décembre 2009, avait déploré l'absence de prise en charge des condamnés soumis à une détention de sûreté, cette carence constituant l'un des éléments qui avaient entraîné la condamnation de l'Allemagne. De même, dans l'arrêt Murray c. Pays-Bas du 26 avril 2016, elle avait conclu au caractère incompressible de facto de la perpétuité infligée au requérant, car l'absence de soins pendant sa détention ne lui avait pas permis d'évoluer et d'envisager une sortie (CEDH, 26 avr. 2016, Murray c. Pays- Bas, [GC], no 10511/10 : Revue de droit pénal, 2016, comm. 120). Là encore, l'inexistence d'une prise en charge avait donné lieu à une violation. On rapprochera enfin de ce mouvement prétorien l'arrêt de la chambre criminelle du 28 mars 2018 cassant l'arrêt d'une juridiction nationale de la rétention de sûreté. Celle-ci avait confirmé la décision d'une juridiction régionale qui ne comprenait aucun motif sur la nature de la prise en charge, médicale, sociale et psychologique dont avait pu bénéficier le condamné au cours de l'exécution de sa peine (Cass. crim., 28 mars 2018, n 17-86938). La réalité du suivi du condamné est alors devenue une condition de fond de l'application de la rétention de sûreté, à la suite de la modification de l'article 706-53-14 du Code de procédure pénale par la loi du 10 août 2010 (tirant les leçons de la condamnation de l'Allemagne dans l'arrêt du 12 décembre 2009). 
En résumé, l'existence d'une prise en charge du condamné - et le constat de son échec - est seule de nature à justifier l'application d'une mesure de sûreté, par essence subsidiaire. Or, en l'espèce, aucun suivi de nature, non pas à soigner, mais à réinsérer le condamné, n'avait été prévu.

Enfin, toute mesure de sûreté ne devant être prononcée qu'en dernier recours, il était donc également peu pertinent d'y soumettre un condamné qui aurait été condamné à un emprisonnement assorti d'un sursis, celui-ci pouvant, de surcroît, ne pas être probatoire : pourquoi aurait-on pu décider de le surveiller à la fin de sa peine, la juridiction de jugement, préalablement intervenue, n'ayant pas jugé bon de le soumettre à une probation (dont la mesure reprenait les obligations et interdictions) ? La question se posait d'autant plus que lorsqu'un juge prononce une peine avec sursis (même partiel), c'est donc le signe que le comportement reproché n'est pas si grave : une surveillance $a$ posteriori n'aurait donc pas été justifiée, signe que la mesure n’était pas proportionnée.

Ce nouveau dispositif était-il disproportionné ?

En prévoyant que la durée de la mesure était fonction, non de la peine prononcée, mais de la peine encourue, le législateur prenait effectivement le risque d'une censure. Une personne condamnée au minimum requis de cinq ans d'emprisonnement (ou trois en récidive), éventuellement avec sursis, pouvait très bien être l'objet d'une surveillance pendant une durée maximale de 10 ans en cas de crime ou de délit passible de 10 ans d'emprisonnement. Enfin, le renouvellement de la mesure pouvait être décidé aux mêmes conditions que la décision initiale, sans qu'il soit exigé que la dangerosité de la personne soit corroborée par des éléments nouveaux ou complémentaires, ce qui allait, là encore, à l'encontre des principes en la matière : par exemple, chaque renouvellement des MICAS, au bout d'une durée cumulée de six mois, est subordonné à l'existence d'éléments nouveaux (CSI, art. L. 228-1).

Reste à déterminer la portée de cette décision. Il ne s'agit certainement pas d'une remise en cause de la technique de la mesure de sûreté qui, bien que décriée, n'a cessé de se développer au cours des dernières décennies, ni même d'une censure de la volonté législative de créer un statut spécial du terroriste en matière de peine (la loi du 3 juin 2016 a en effet considérablement durci les conditions d'octroi de la libération conditionnelle des auteurs d'infractions terroristes cependant que celle du 21 juillet 2016 les a exclus du bénéfice de la suspension et du fractionnement de peine, de la semi-liberté et du placement à l'extérieur et des crédits de réduction de peine ; sur le sujet, E. Bonis, «À propos de l'article 8 de la loi no 2016- 987 du 21 juillet 2016 : vers la création d'un droit spécial des aménagements de peine pour les condamnés pour terrorisme », Dr. pén. 2016, étude 26 ; P. Poncela, « Peines et prisons : la régression. À propos des lois du 3 juin et 21 juillet $2016 »$, RSC 2016, p.565 et s.).

Doit-on alors y voir, plus ponctuellement, la censure d'un texte qui n'a pas pris les précautions élémentaires lui évitant de passer sous les Fourches caudines du Conseil constitutionnel (rapp. Cons. const., déc. $\mathrm{n}^{\circ}$ 2020-801 DC du 18 juin 2020 à propos de la loi visant à lutter contre les contenus haineux sur internet) ? 